\title{
R2D2: A Region-Based Recursive Doppler Dealiasing Algorithm for Operational Weather Radar
}

\author{
Monika Feldmann, ${ }^{\mathrm{a}, \mathrm{b}, \mathrm{d}}$ Curtis N. JAmes, ${ }^{\mathrm{c}, \mathrm{d}}$ MArco Boscacci, ${ }^{\mathrm{b}}$ Daniel Leuenberger, ${ }^{\mathrm{b}}$ Marco Gabella, \\ Urs Germann, ${ }^{\mathrm{b}}$ DANIEl Wolfensberger, ${ }^{\mathrm{a}, \mathrm{b}}$ AND AleXis Berne ${ }^{\mathrm{a}}$ \\ ${ }^{a}$ Remote Sensing Laboratory, École polytechnique fédérale de Lausanne, Lausanne, Switzerland \\ ${ }^{\mathrm{b}}$ MeteoSwiss, Zurich, Switzerland \\ ${ }^{\mathrm{c}}$ Embry-Riddle Aeronautical University, Prescott, Arizona
}

(Manuscript received 17 April 2020, in final form 10 October 2020)

\begin{abstract}
Region-based Recursive Doppler Dealiasing (R2D2) is a novel dealiasing algorithm to unfold Doppler velocity fields obtained by operational radar measurements. It specializes in resolving issues when the magnitude of the gate-to-gate velocity shear approaches or exceeds the Nyquist velocity. This occurs either in highly sheared situations, or when the Nyquist velocity is low. Highly sheared situations, such as convergence lines or mesocyclones, are of particular interest for nowcasting and warnings. R2D2 masks high-shear areas and adds a spatial buffer around them. The areas between the buffers are then identified as continuous regions that lie within the same Nyquist interval. Each region subsequently is assigned its most likely Nyquist interval by applying vertical and temporal continuity constraints, as well as supplemental wind information from an operational mesoscale model. The shear zones are then resolved using $2 \mathrm{D}$ continuity in azimuth and range. This $4 \mathrm{D}$ procedure is repeated until no further improvement can be achieved. Each iteration with fewer folds identifies fewer but larger continuous regions and less shear zones until an optimum is reached. Residual errors, often related to shear greater than the Nyquist velocity, are contained to small areas within the buffer zones. This approach maximizes operational performance in high-shear situations and restricts errors to minimal areas to mitigate error propagation.
\end{abstract}

KEYWORDS: Wind shear; Wind; Radars/Radar observations; Remote sensing; Error analysis

\section{Introduction}

\section{a. Doppler radar challenges in complex terrain}

Doppler velocity measurements are a useful weather monitoring tool, as they provide near-real-time information about the inner dynamics of meteorological phenomena. The identification of strong gate-to-gate velocity shears in azimuth (corresponding to rotation), or in range (corresponding to divergence), can provide key information about meteorological hazards such as turbulence, severity of convection, frontal activity, and wind gusts. For nowcasting applications and weather warnings, as well as data assimilation, the correct retrieval of Doppler velocity measurements is essential.

As the Doppler velocity corresponds to the measured phase shift between the incident and reflected signal, ambiguous measurements occur if the shift exceeds $2 \pi$. This phenomenon is called aliasing. The maximum unambiguous velocity, or Nyquist velocity, $\pm v_{\mathrm{NYQ}}$, increases with increasing pulse repetition frequency (PRF) and thus results in a trade off with maximum unambiguous range. Since Doppler velocity measurements provide a valuable tool in analyzing the dynamical evolution of precipitation systems and can aid in assessing potential hazards in weather situations, a high-quality reconstruction of the true velocity field is essential. The process of removing the velocity folds, or aliases, is called dealiasing.

\footnotetext{
${ }^{\mathrm{d}}$ These authors were the main contributors to this work.
}

Corresponding authors: Monika Feldmann, monika.feldmann@ epfl.ch; Curtis N. James, curtis.james@erau.edu
In mountainous terrain, the Doppler dilemma presents an additional challenge. With beam blockage from terrain significantly reducing the observable terrain, a long unambiguous range from multiple radars can help ensure more accurate measurements, by covering the same area from different directions and angles (Germann et al. 2016a). Moreover, the rapid, dynamic nature of mountain meteorology calls for a frequent volume update to adequately monitor evolving precipitation fields (Germann et al. 2016a). In this study, we focus on the operational radar network of MeteoSwiss Rad4Alp (Germann et al. 2015, 2016b) that is tailored to Alpine precipitation monitoring. With long ranges, particularly at low elevations, and a rapid volume update over 20 elevations it is well suited to the operational requirements of precipitation, thunderstorm, and hail nowcasting. Due to limited observable range and construction constraints in an Alpine environment, the network consists in five polarimetric C-band radars.

These decisions result in an overall low Nyquist velocity, which introduces a particularly challenging situation for handling Doppler velocity measurements. Problems arise when turbulence, shears, or rotational patterns commonly present in the airflow around the Alps are not well resolved by the Nyquist velocity.

\section{b. Existing dealiasing methods}

The main feature of many dealiasing algorithms is 2D spatial continuity within each elevation scan (He et al. 2012; Witt and Brown 2009; Zhang and Wang 2006; Eilts and Smith 1990). Here, each gate is inspected with respect to its velocity difference with its neighbors, to detect and resolve aliasing. This also increases the dependency of dealiasing decisions on single 
gates. Helmus and Collis (2016), Xu et al. (2011), and Wüest et al. (2000) reduce the importance of single gates by relying on larger areas of information for dealiasing decisions. A regional approach is also taken by Yuan et al. (2020), where areas of contiguous data coverage are first identified and then further split along velocity folds into regions. Each region is assigned a single dealiasing solution, thus also avoiding issues from single gates. A mixture of regional and spatial dealiasing is pursued by Louf et al. (2020). The main dealiasing approach is similar to spatial dealiasing and solves gate by gate; however, it draws on a larger, predefined region around each gate to obtain a solution.

When Doppler velocity cells are disconnected from each other by regions with no echoes, it is often advantageous to utilize supplemental information about the velocity field during the dealiasing process. To estimate the general velocity direction and magnitude above a radar, the velocity-azimuth display (VAD) is a method that assumes a horizontally uniform velocity field and provides an estimated vertical profile of horizontal velocity (Germann 1999). At constant altitude, all available radar measurements are fitted to a sine curve with azimuth to estimate velocity magnitude and bearing from the phase and amplitude of the sine curve.

While different methods exist to derive a VAD (Germann 1999; Tabary et al. 2001; Xu et al. 2010), several dealiasing approaches rely on the VAD as additional wind information to aide dealiasing (He et al. 2012; Xu et al. 2011; Witt and Brown 2009; Wüest et al. 2000; Eilts and Smith 1990). The VAD provides useful information to initialize dealiasing; however, it does not represent spatial heterogeneity and requires sufficient data coverage around the radar in order to create a representative velocity profile. Therefore a VAD is inadequate for initializing dealiasing when spatial heterogeneity of the velocity field and/or high shear exist in the radial velocity field. Zhang and Wang (2006) and also Louf et al. (2020) propose to identify the primary wind direction without additional wind information, by initializing close to the zero isodop. This can be problematic in cases, when wind velocities exceed multiples of the Nyquist interval, thus leading to the occurrence of several zero isodops. James and Houze (2001) proposed the use of 4D data continuity, leveraging the vertical and temporal dimensions available in operational Doppler data streams, to improve dealiasing in the presence of shear or heterogeneity. Yuan et al. (2020) use local reference points from the current sweep, or from the sweeps above and from a previous radar sweep if necessary, to determine the general flow. Thus, Yuan et al. (2020) have also extended dealiasing from 2D to 4D continuity constraints. Louf et al. (2020) utilize 4D continuity within their error detection procedure. Lim and Sun (2010) showed that using the product of assimilated Doppler velocities into a numerical weather prediction (NWP) model yields a substantially better approximation of the velocity field, retaining spatial heterogeneity. Xu et al. (2013) also make use of hourly model-predicted winds to improve velocity estimates for isolated areas at far ranges.

The preceding techniques do not specifically target problematic dealiasing phenomena, such as supercellular mesocyclones or tropical cyclones, though some recent studies have focused on targeting these specifically, such as $\mathrm{Xu}$ and Nai (2017) for mesocyclones and Jiang and Xu (2016) and Chang et al. (2019) for tropical cyclones. These situations are both characterized by strong vortex winds that pose difficult dealiasing problems due to the curvature of the velocity field and high shear. These latter studies propose fitting vortex models to the radius and magnitude of the rotational velocity maxima and reinitializing dealiasing with the fitted vortex model.

Recent work has shown an aliasing-robust implementation of the integrated velocity-azimuth process (IVAP) (Liang et al. 2019). This method effectively provides localized reference winds and thus aides local turbulence phenomena, however, is computationally quite demanding.

Since 2005, MeteoSwiss has implemented four-dimensional dealiasing (4DD; James and Houze 2001) as its operational dealiasing algorithm. 4DD specializes in solving dealiasing problems with low Nyquist velocities, as mentioned above, by applying 4D continuity constraints from the previous time step, the elevation above, and in 2D space within the targeted elevation. If there is insufficient information in the sweeps before and above, it relies on the VAD (Tabary et al. 2001) as a last resort to initialize dealiasing. The particular challenge with low Nyquist velocities is the occurrence of multiple folds. This can make it difficult to identify the correct zero isodop without additional information.

4DD initializes dealiasing in single velocity gates that have a high level of agreement with the according gate in the sweep before and above. From there, the algorithm spreads, applying $2 \mathrm{D}$ continuity constraints. This procedure is repeated until the majority of the data has been reconstructed. Remaining data segments are targeted with a windowing technique, where a larger spatial window is used to estimate the correct Nyquist interval of remaining, uncorrected areas.

Particularly in stratiform precipitation situations, where the data coverage is high and velocities are more homogeneous, 4DD provides very reliable results. Stratiform events constitute the majority of precipitation situations in Switzerland, especially during the cold season.

However, in convective situations with low Nyquist velocity, when the data coverage is more fragmented and strong gate-togate shears and turbulence abound, 4DD exhibits some performance issues. If dealiasing erroneously initializes in a noisy gate, the error propagates in space via the $2 \mathrm{D}$ spatial dealiasing technique and feeds into the sweeps below and the next time steps due to the vertical and temporal continuity constraints. The same issue occurs in the case of unresolved wind shear from one gate to the next. Spatial dealiasing can assign the wrong Nyquist interval in these situations, resulting in a spreading error. Additionally, the VAD method has issues in low data coverage, as it reconstructs velocity magnitude and direction from measurements at constant altitude around the radar. As the VAD assumes spatial homogeneity of the wind field, it is also less representative in heterogeneous, turbulent situations, as commonly found in convection. The complex wind field often observed in the Alps, especially in the presence of deep convection, further complicates the dealiasing problem.

Therefore, we focus this study on developing a technique that reduces the importance of single gates on dealiasing 


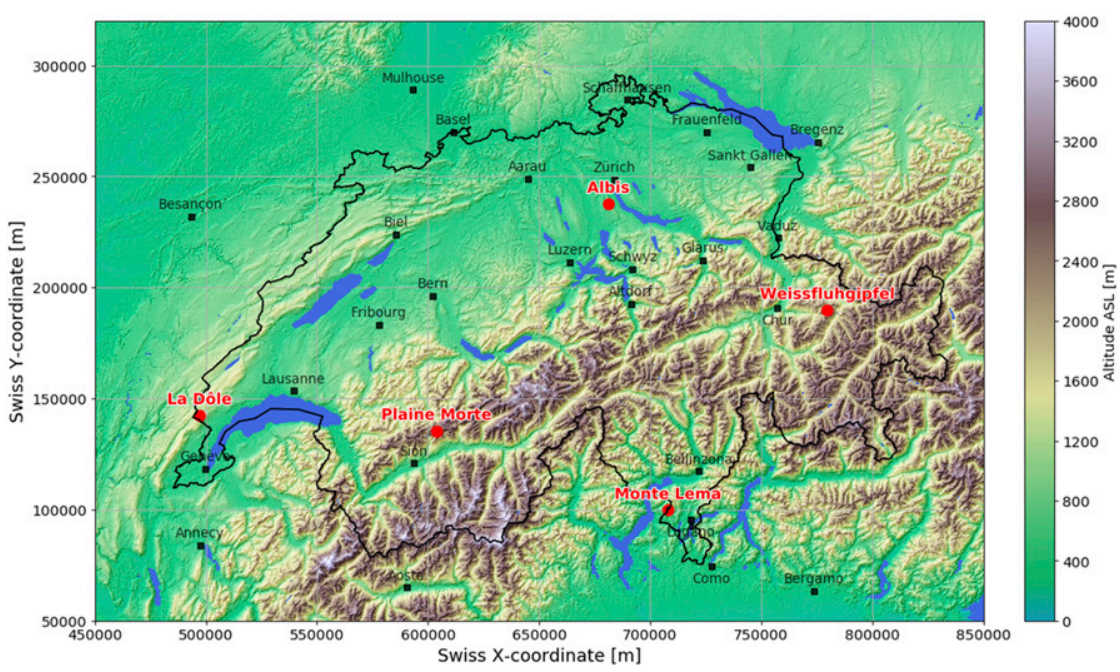

FIG. 1. Elevation map of the western Alps with the five radar sites (marked in red) of the dual-polarization operational Swiss weather radar network (Rad4Alp). Major cities are indicated in black.

decisions and mitigates error propagation horizontally, but also vertically and in time.

This paper describes the development of a novel dealiasing algorithm targeted at these challenging circumstances. Section 2 introduces the data that were used for algorithm development and testing. Section 3 describes the design of the new algorithm, called Region-based Recursive Doppler Dealiasing (R2D2). In section 4, the results of a comparison between R2D2 and its predecessor 4DD are presented. Finally, section 5 concludes the evaluation of R2D2.

\section{Data}

\section{a. Radar network of Switzerland}

This dealiasing approach aims to improve over the current operational algorithm of Switzerland's operational radar network. The radar network of MeteoSwiss consists of five polarimetric C-band radars (Germann et al. 2016a; Bundesamt für Meteorologie und Klimatologie MeteoSchweiz 2018). Their altitudes range from 900 to $3000 \mathrm{~m}$ above mean sea level (MSL), which ensures a good coverage of the Swiss plains and above the Alps. They are located on the peaks of Albis, La Dôle, Monte Lema, Pointe de la Plaine Morte, and Weissfluh (see Fig. 1).

\section{1) Operational ScAn StRategy}

The radars are synchronized and operate on the same scan strategy. The scan strategy is static and consists of 20 tilts ranging from $-0.2^{\circ}$ to $40^{\circ}$ with corresponding maximum unambiguous ranges between 246 and $27 \mathrm{~km}$. Correspondingly, the Nyquist velocity ranges from 8.25 to $20.63 \mathrm{~m} \mathrm{~s}^{-1}$. The elevations are interleaved, with slightly higher pulse repetition frequencies and thus higher Nyquist velocities (with an increment $\sim 1.5 \mathrm{~m} \mathrm{~s}^{-1}$ ) stacked in between slightly lower ones. The entire volume is updated every $5 \mathrm{~min}$. The interleaved nature allows for a partial volume update of 10 elevations every $2.5 \mathrm{~min}$. The data are stored at $1^{\circ}$ azimuthal and $500 \mathrm{~m}$ range resolution, here referred to as gates.

Achieving this high temporal resolution at all elevation angles requires a high rotation rate, limiting the possible number of pulses per azimuth degree. To avoid issues with noise from too few pulses, each elevation scan uses a single pulse repetition frequency.

The long ranges at low elevations allow for good coverage above the complex terrain in Switzerland, with different radars observing areas from different perspectives. The fast update rate is tuned to provide timely observations on rapidly evolving precipitation systems in orographic and convective situations.

The Doppler velocity fields operationally available are processed with 4DD, as described in section 2, using each corresponding VAD as an environmental wind estimate.

\section{2) Velocity-AZIMUTH DisPlay}

VADs for all five radars are operationally produced every $5 \mathrm{~min}$. The implemented algorithm follows Tabary et al. (2001) and is briefly described in the following section.

The VAD utilizes the sinusoidal behavior of a Doppler velocity field at constant range. By merging all available velocity measurements at constant altitude from the two highest elevation scans, the VAD algorithm reconstructs a vertical profile of horizontal wind above the radar. First the VAD algorithm is also faced with aliased velocity gates. To solve this, the azimuthal derivative of the radial wind is computed. As the derivative of the radial wind has sinusoidal behavior, a sine curve is fitted to the derivative as a first estimate of the VAD wind vector. Thereafter, this first estimate is used to dealias all velocity gates. Then, a second sine curve is fitted to the dealiased velocity gates. The phase and amplitude of this sine curve yield the VAD wind vector if sufficient data are available. The elevations at $35^{\circ}$ and $40^{\circ}$ represent and area of approximately $22 \mathrm{~km}$ around each radar. Fitting a sine-curve assumes a 
horizontally uniform velocity field within this region. Low data coverage and turbulent situations compromise the quality of the vertical profile.

\section{b. COSMO-1 numerical weather prediction model}

COSMO-1 is the operational, high-resolution NWP model of MeteoSwiss with a horizontal mesh size of $1.1 \mathrm{~km}$ (de Morsier et al. 2012). It provides a high-resolution 3D wind field over the Swiss radar domain and is used here as an alternative source of environmental wind information.

As a nonhydrostatic, convection-permitting model it also partially resolves the internal dynamics of convective systems. To reproduce the observed wind field as closely as possible, COSMO-1 is used in analysis mode, where it assimilates observations from radiosondes, surface weather stations, wind profilers, aircrafts and surface precipitation estimates from the five Swiss C-band radars. The boundary conditions are provided by the European Centre for Medium-Range Forecasts (ECMWF) global model. To match the temporal resolution of the radar data, the output frequency is set to $5 \mathrm{~min}$.

\section{c. Forward polarimetric radar operator}

A forward polarimetric radar operator, as introduced in Wolfensberger and Berne (2018), allows us to transform COSMO-1 output into the perspective of a radar. The radar operator is configured to mimic the Swiss operational scan strategy, replicating the maximum range, Nyquist velocity, and elevation angles at the five radar locations. It additionally accounts for terrain blockage.

Our main interest is the creation of a simulated Doppler velocity field for testing the performance of dealiasing algorithms. With the possibility to change the Nyquist velocity easily, the COSMO-1 data are processed once with the operationally implemented Nyquist velocity and once without any Nyquist constraint. This allows the computation of a ground truth, to which the folded velocity field and different dealiasing results can be compared.

\section{d. Analyzed case studies}

The analyzed data expand over six case studies representing different meteorological situations. With the focus being on improving the dealiasing performance in convective situations, four of them represent different convective cases, while the other two represent stratiform situations. The selected cases present challenging dealiased situations due to the presence of high wind speeds, low data coverage, and high wind shear. Table 1 provides an overview of the case studies.

All case studies provide at least $3 \mathrm{~h}$ of data for the analysis, with sufficient previous data to allow for algorithm startup. Overall, 372 time steps are analyzed, resulting in 7440 elevation scans.

\section{R2D2}

\section{a. First guess field}

Instead of ingesting the radar's VAD as additional environmental wind information, we replace it with NWP data. For this purpose we use COSMO-1 analysis' 5 min output of the 3D
TABLE 1. Case studies.

\begin{tabular}{|c|c|c|}
\hline Date & $\begin{array}{l}\text { Observing radar } \\
\text { location }\end{array}$ & Event \\
\hline 2 Aug 2017 & Albis & Supercellular convection \\
\hline 11 Jun 2018 & La Dôle & Multicellular convection \\
\hline 15 Jun 2019 & La Dôle & Mesoscale convective system \\
\hline 11 Aug 2019 & Plaine Morte & Supercellular convection \\
\hline 7 Mar 2019 & Weissfluhgipfel & $\begin{array}{l}\text { Cold front passage with } \\
\text { high winds }\end{array}$ \\
\hline 19 Oct 2019 & Monte Lema & $\begin{array}{l}\text { Cold front passage with } \\
\text { collapsing Foehn situation }\end{array}$ \\
\hline
\end{tabular}

wind field. To avoid errors stemming from convective features, such as thunderstorm inflow, gust fronts, or mesocyclones that may be misplaced in space and time, the field is smoothed with a $20 \mathrm{~km}$ convolution kernel. Dynamical convective features generally have a size smaller than $10 \mathrm{~km}$ (Markowski and Richardson 2010). By applying the convolution to the wind field, the general flow and spatial heterogeneity are conserved, while strongly reducing the influence of convective features.

To derive a first guess of the wind field from the radars' perspective, the wind field is transformed to a radial wind field for each radar using a purely trigonometric approach. The radial wind field is extracted at all 20 elevations. This COSMOderived radial wind estimate for each elevation replaces the VAD in our tests.

The first-guess field is only used if there are no other data sources available. Its intention is to provide the general direction and magnitude of the flow and its spatial distribution, not small-scale dynamic features.

\section{b. Dealiasing procedure}

Considering the existing algorithms described in section 2 , our main criteria for a novel algorithm can be summarized as follows:

1) Apply a regional approach to reduce importance of single gates.

2) Utilize 4D continuity to reduce dependency on environmental winds.

3) Mitigate error propagation.

4) Supplement dealiasing using 3D velocity information.

5) Ensure reliability and efficiency for operational implementation.

Following James and Houze (2001), R2D2 best operates on a continuous data stream, where the algorithm can leverage 4D constraints. In each volume, it starts at the highest elevation where the Nyquist velocity is typically larger and where Doppler velocity data are generally less noisy, then proceeds downward to the more difficult low-elevation sweeps. Within each sweep, it follows a nine-step procedure, as shown in Fig. 2.

\section{1) DAtA Filtering: STEP 1}

Prior to dealiasing, the operational clutter detection algorithm of MeteoSwiss is applied. It makes use of criteria on reflectivity, Doppler spectrum and velocity, signal fluctuations, differential phase shift and reflectivity, copolar correlation coefficient, and dynamic and static clutter maps to 


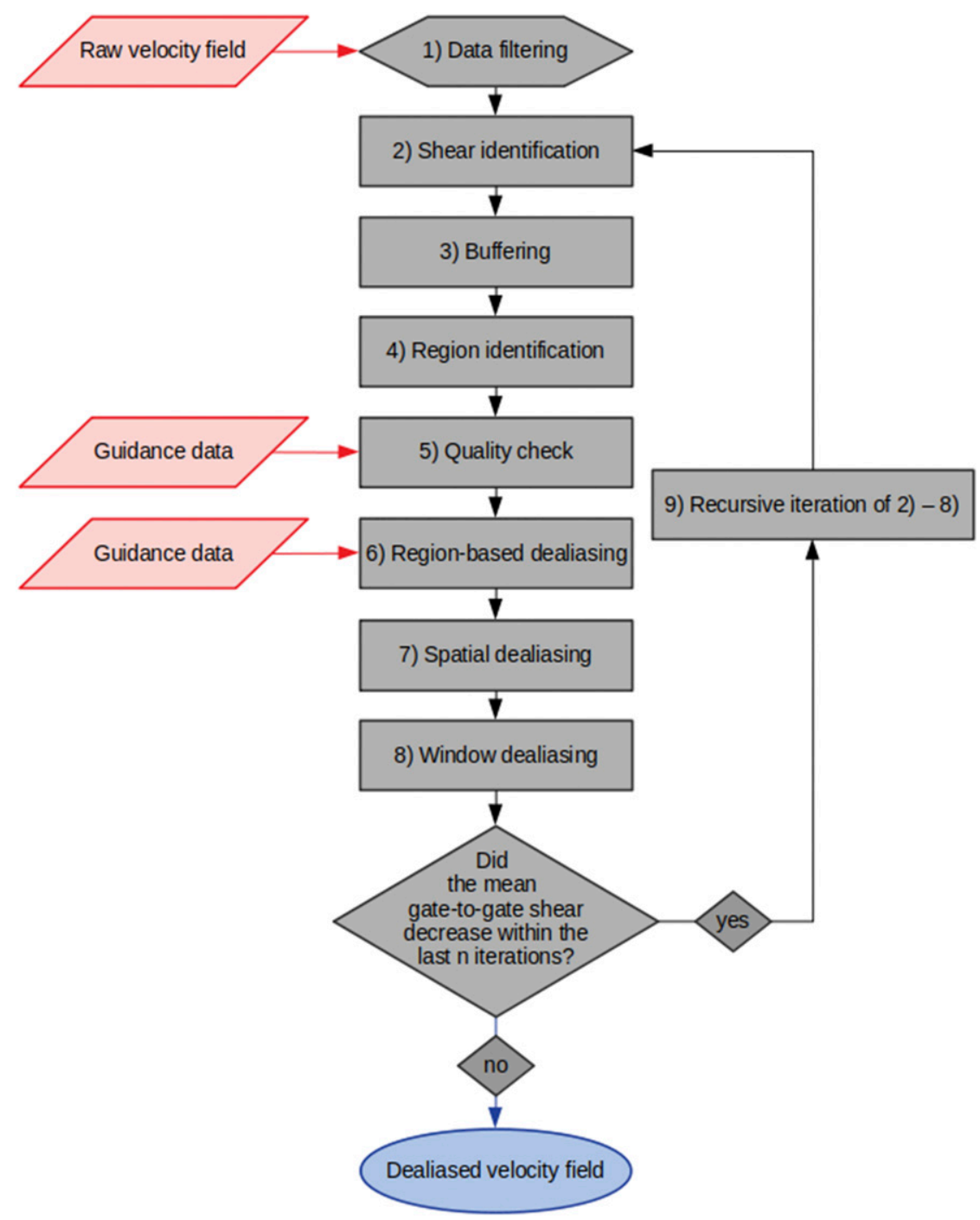

FIG. 2. Flowchart of algorithm steps within R2D2. Red corresponds to input data, gray to algorithm step, and blue to output data.

identify nonmeteorological echoes. Clutter cancellation is done individually for each $83 \mathrm{~m}$ gate, the highest possible radial resolution, which is an important aspect in complex terrain (MeteoSwiss 2020; Germann and Joss 2004). Faced with the challenge of retaining as much data as possible, while dealing with considerable terrain contamination in the main and side lobes of the radar beams, the resulting Doppler velocity field still contains noise and residual clutter.

Therefore, in the first step, the raw radial velocity data are filtered to minimize the influence of noise, which can introduce errors during dealiasing. The algorithm first deletes all small, isolated echoes or speckles in each sweep that occupy an area equivalent to or less than 10 connected gates at a range of $100 \mathrm{~km}$ from the radar, and whose velocity values are close to zero. Experience has shown that these small regions generally contain noise and would otherwise lead to problems during the windowing procedure (see step 8). In addition, the user may opt to apply a Bergen and Albers filter (Bergen and Albers 1988), to remove problematic gates on the edges of larger data regions, or apply a reflectivity threshold that discards all gates below a certain value. Finally, the user has the ability to remove terrain clutter. If the radar's visibility (Gabella et al. 2017) decreases with range along a radial, or drops below a user-defined threshold, the surrounding gates may be discarded as shadowed regions, respectively. The filtering process seeks to improve the quality of the raw velocity data and increase the likelihood of success in a simple and efficient manner, though too much deletion (especially of terrain shadowed regions) can create discontinuities in the data and make dealiasing more challenging.

\section{2) ANOMALOUS SHEAR IDENTIFICATION AND BUFFERING: STEPS 2 AND 3}

Before initializing dealiasing, the maximum value of the absolute difference $D$ of each velocity gate to its up to eight 
neighbors is computed. All gates where the maximum difference exceeds a primary threshold $b$ are flagged as high-shear gates. All gates within a $5 \times 5$ range of the initially identified gate are also flagged. These buffer zones covering high-shear areas and their vicinity are excluded from the initial dealiasing attempts. They are deemed to be difficult to dealias, as they either contain a velocity fold or high wind shear. The size of $5 \times 5$ gates aims to include small regions of unresolved shear lying close to detectable shear, without making the zones so large that they cover excessive amounts of data.

The conceptual schematic in Fig. 3 exemplifies this procedure. Figure 3 a shows the initial velocity field that contains a turbulent region on the left and a velocity fold at the bottom right. Figure $3 \mathrm{~b}$ depicts the maximum gate-to-gate difference in any direction from each gate, which strongly highlights the fold, but also the area of turbulence. Figures $3 \mathrm{c}$ and $3 \mathrm{~d}$ then show the resulting buffered areas in gray, overlaid on the velocity and maximum difference field, respectively. Here, a threshold of $8 \mathrm{~m} \mathrm{~s}^{-1}$ was used, which corresponds to $0.8 v_{\mathrm{NYQ}}$.

\section{3) LOW-SHEAR REGIONS AND QUALITY CHECK: STEPS 4 AND 5}

After the buffer zones have been flagged, it is assumed that the remaining regions do not contain any folds or high shear. Each congruent region is referenced with an ID and assigned to the same Nyquist interval. Figure 4 illustrates a modeled example of this procedure. Figure 4 a shows the folded velocity as produced by the radar operator, noting that by Swiss convention positive radial velocity means inbound motion. Figure $4 \mathrm{~b}$ depicts the maximum difference field. The resulting buffer zones, derived by the same procedure as in Fig. 3, are shown in dark blue in Fig. 4c. All contiguous white regions are assigned an individual ID for the subsequent dealiasing procedure.

As we are dealing with very small Nyquist velocities, there remains the possibility of an extended region of unresolved shear, a hidden fold, where the difference to the neighboring gates actually exceeds the Nyquist velocity, inside the identified regions. To prevent this from happening, a quality check is implemented. Each region is compared to the corresponding gates in the sweep above, the sweep before and the first guess field. These three fields provide the guidance information for each region. If the standard deviation of the difference between all gates in a region and its guidance data exceeds a certain threshold $g$, it is likely that there still remains a fold in the region, or its guidance data. In this case, the region is also flagged as a buffer zone to avoid introducing or propagating a large error. By comparing each region to the guidance, the algorithm makes use of 4D continuity information available in the velocity field. As the elevations are relatively close to each other and are updated every $5 \mathrm{~min}$, in most cases they correspond to the velocity field of the current elevation very well.

\section{4) Dealiasing: Steps 6-8}

The remaining regions are then assigned into their respective Nyquist intervals. To obtain the correct Nyquist interval, each region as a whole is compared to the corresponding gates
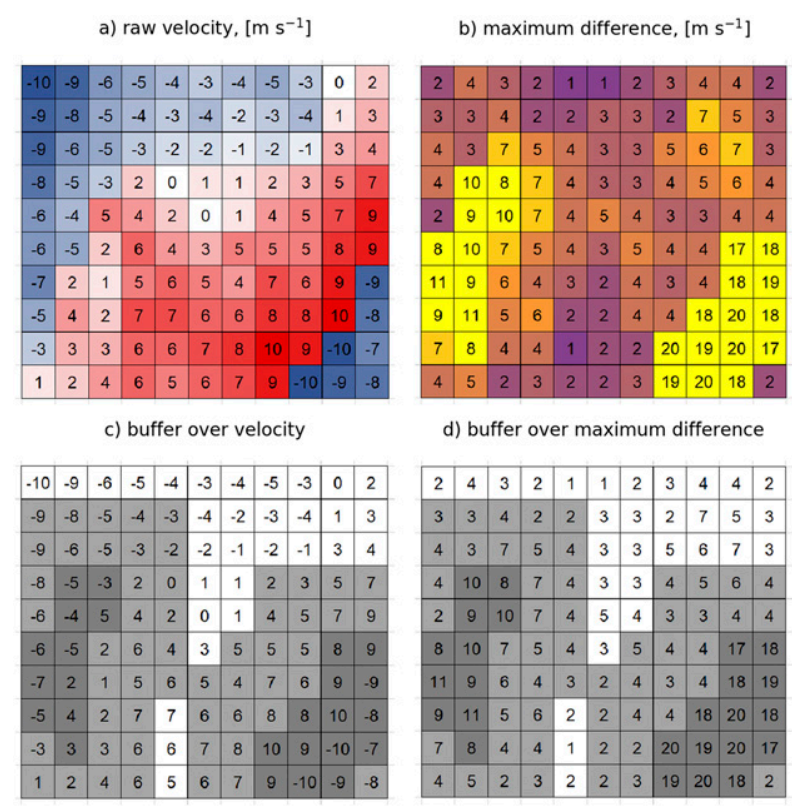

d) buffer over maximum difference

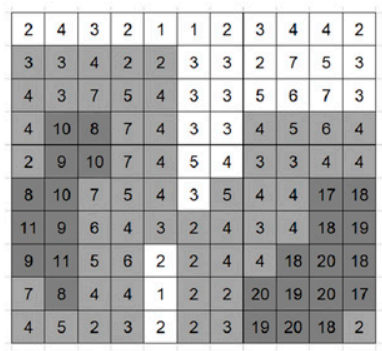

FIG. 3. Conceptual schematic of buffering procedure of R2D2: (a) $v_{\mathrm{DOP}}\left(\mathrm{m} \mathrm{s}^{-1}\right), v_{\mathrm{NYQ}}=10 \mathrm{~m} \mathrm{~s}^{-1}$; (b) maximum difference $D$ $\left(\mathrm{m} \mathrm{s}^{-1}\right.$ ), with a threshold $b=0.8 v_{\mathrm{NYQ}}=8 \mathrm{~m} \mathrm{~s}^{-1}$; (c), (d) $v_{\mathrm{DOP}}$ and $D$ with gates, where $D \geq b$, in dark gray shading and the resulting $5 \times 5$ buffer in light gray shading.

in the sweep above and in the previous time step. In the first iteration, if both of these data are missing in more than $90 \%$ of the gates, the first guess field is used as an added constraint. In subsequent iterations, all guidance information is used. For each guidance region, the average difference to the current region is computed and the Nyquist interval of least absolute difference is assigned. If the Nyquist interval matches for all available guidance information, the region is dealiased. If there is disagreement, the region is flagged as a buffer zone and reserved for spatial dealiasing.

After all regions have been dealiased in this manner, spatial dealiasing corrects the buffer zones. Spatial dealiasing follows the procedure of James and Houze (2001), except that it is only used within these buffer regions in order to confine potential errors to smaller areas. Gates adjoining dealiased gates are corrected by shifting them by a number of Nyquist intervals to result in a velocity difference smaller than a certain threshold $s$ to the dealiased neighbors. This procedure is repeated in different scanning directions up to $n$ times, with iteratively relaxing thresholds $s_{n}$. This allows regions with small velocity differences to be dealiased first and strong shear zones are targeted last. This approach mitigates error propagation from high shear areas. By applying spatial dealiasing only within buffer zones, the dependency on single gates is limited to the buffer zones. Errors resulting from noisy or high-shear gates will thus also be confined to the buffer zones. Propagation of errors is avoided by surrounding buffered gates with the previously dealiased regions.

Remaining data that have not been corrected by spatial dealiasing are then targeted by windowing as in James and Houze (2001), where a larger spatial window, i.e., 
a) raw vel, $\left[\mathrm{m} \mathrm{s}^{-1}\right]$

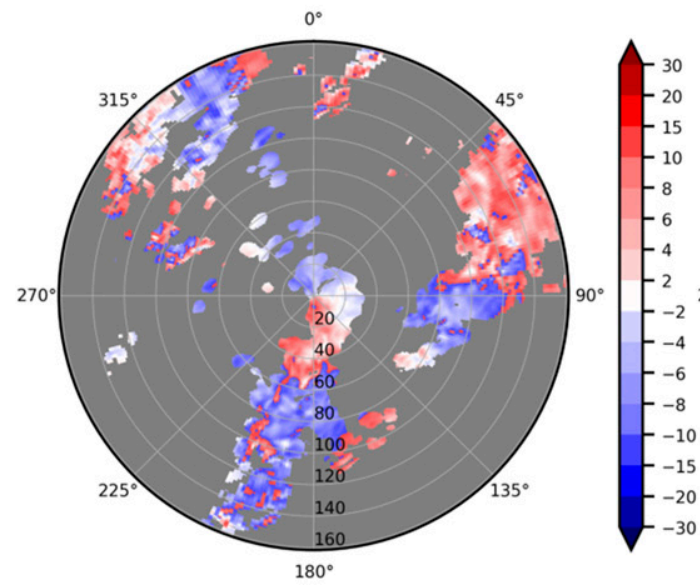

c) buffer zones 1. IT

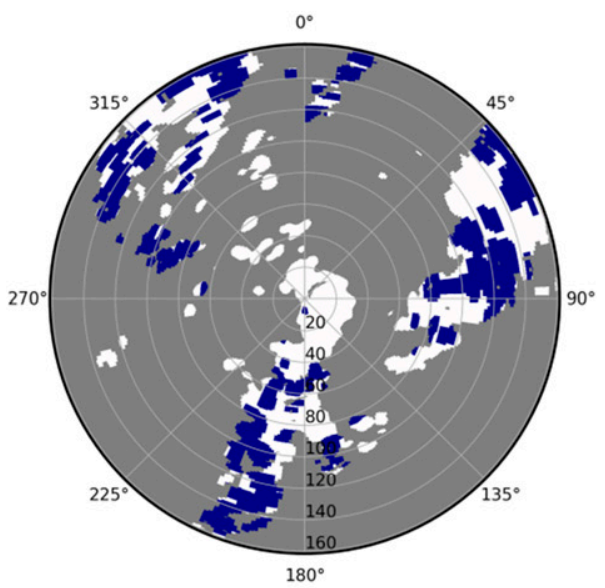

e) dealiased vel, $\left[\mathrm{m} \mathrm{s}^{-1}\right]$

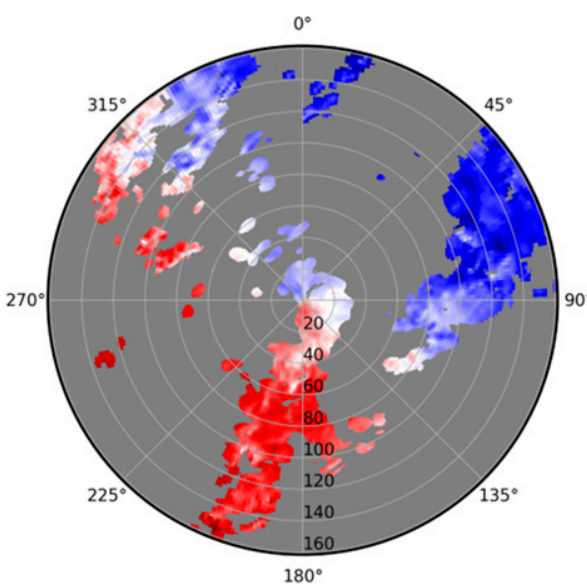

b) max difference $1 . \mathrm{IT},\left[\mathrm{m} \mathrm{s}^{-1}\right]$

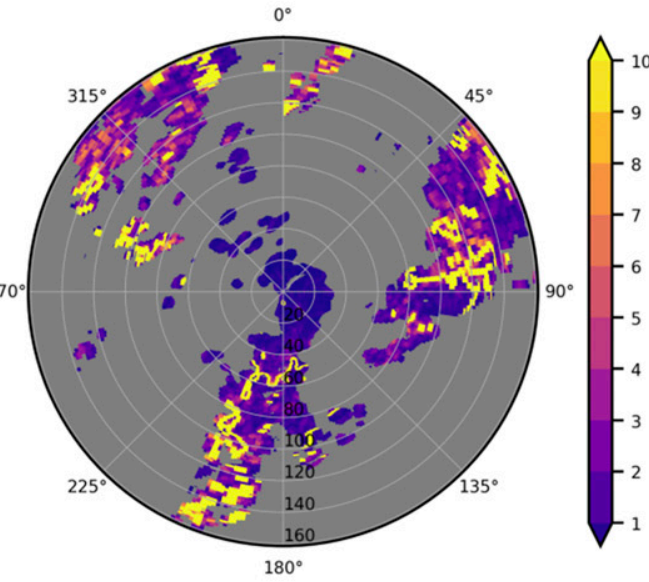

d) buffer zones last IT

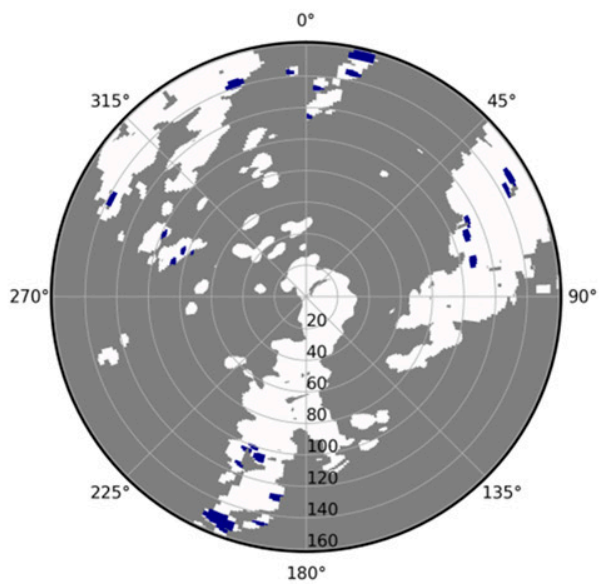

f) true vel, $\left[\mathrm{m} \mathrm{s}^{-1}\right]$
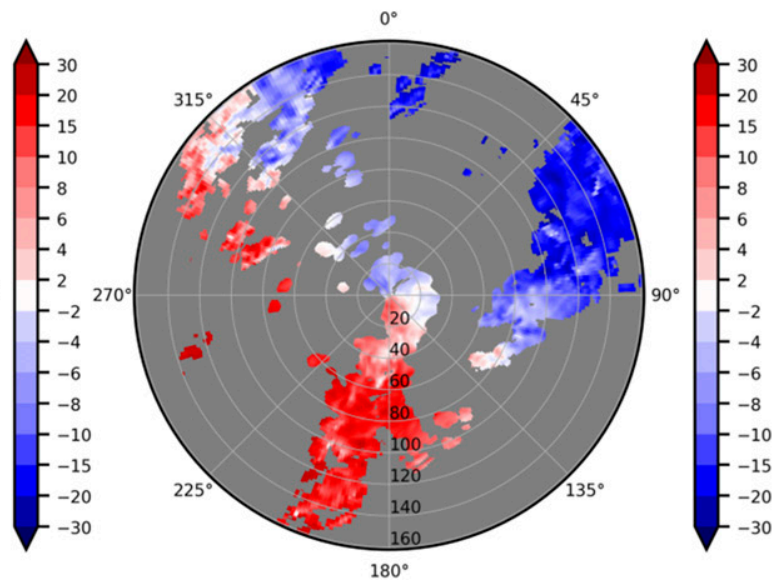

FIG. 4. Region identification of R2D2, modeled data; $v_{\mathrm{NYQ}}=9.6 \mathrm{~m} \mathrm{~s}^{-1}, b=0.8 v_{\mathrm{NYQ}}=7.7 \mathrm{~m} \mathrm{~s}^{-1}$ : (a) modeled folded velocity, (b) maximum difference $D$, (c),(d) buffer zones (blue) of first and last (third) iterations, (e) dealiased velocity, and (f) modeled true velocity. 
$10 \times 10$ gates, is used to estimate the Nyquist interval from neighboring dealiased data. This routine allows isolated cells that did not qualify as regions to be dealiased adequately as well.

\section{5) RECURSIVE ITERATION: STEP 9}

After completing a first attempt at dealiasing an elevation sweep, the algorithm recursively iterates again through steps $2-8$. On the second attempt, most folds have already been removed, which results in larger regions and less buffer zones. This can be seen in Fig. 4d, which shows the identified buffer zones of the last iteration before yielding the dealiasing result. The folds have been effectively removed with remaining buffer zones predominantly covering high shear regions. With larger regions, more guidance data are available to assign the correct Nyquist interval, which yields a more robust result. By reducing the number of folds included in buffer zones and thus shrinking their size, the dependency on spatial dealiasing is further reduced.

For each iteration, the average of the maximum absolute difference field $D, D_{\text {av }}$, is computed. As long as $D_{\text {av }}$ decreases within a certain number of iterations $i$, the procedure is repeated, until a minimum value is reached and no further optimization can be found within $i$ iterations. Consequently, in Fig. 4, R2D2 performs five iterations with the optimum being reached after three iterations. Figures $4 \mathrm{~d}$ and $4 \mathrm{e}$ show the buffer zones and dealiasing result of the third iteration. As a comparison, Fig. 4f shows the true velocity field, as initially modeled in COSMO-1 and obtained by the radar operator.

\section{c. Specific properties of $R 2 D 2$}

Whereas spatial dealiasing (James and Houze 2001; He et al. 2012; Witt and Brown 2009; Zhang and Wang 2006; Eilts and Smith 1990) and region-based dealiasing (Yuan et al. 2020; Xu et al. 2011; Wüest et al. 2000) are known concepts and commonly employed, they are usually not used in combination. A recent exception to this is presented by Louf et al. (2020), where multiple spatial dealiasing approaches are combined with using a larger reference region than only immediately neighboring gates, similar to the windowing procedure.

We effectively combine both approaches, exploiting their respective strengths. Region-based dealiasing is a powerful method where little turbulence or shear is present and data coverage is widespread. Dealiasing entire regions at the same time is also computationally more efficient than spatial dealiasing, reserving computing resources where they are needed most. Spatial dealiasing targets high-shear zones beginning from the areas with lower shear and with strict continuity constraints, then slowly relaxing the constraints and progressing to the higher shear areas.

The buffering procedure serves as a method of identifying areas where spatial dealiasing outperforms region-based dealiasing. Moreover, our recursive region-identifying procedure minimizes the number of folds finally targeted by spatial dealiasing, allowing the regions to grow and merge together. This leaves predominantly high-shear regions to spatial dealiasing, while large, continuous velocity regions are solved by region-based dealiasing. Iteratively minimizing the average maximum difference $D_{\text {av }}$ by repeating the dealiasing procedure simplifies the dealiasing problem from one iteration to the next, yielding a more optimal dealiasing outcome.

Initializing dealiasing by utilizing the sweep above and before constrains data continuity in the vertical and in time (James and Houze 2001). Only in cases where there is insufficient information from spatiotemporal continuity, is a reference first-guess field used. This approach effectively mitigates errors in isolated cells or new cells that appear in a sequence of volumes.

In combination, these properties of R2D2 effectively target the challenge posed by small Nyquist velocities in turbulent, sheared, and high-velocity situations, as well as the challenge of data discontinuity in convective events and mountainous terrain.

\section{d. Technical implementation}

This algorithm was developed within the operational framework of MeteoSwiss's existing operational chain. It leverages code from 4DD (James and Houze 2001), the previous operational algorithm. The algorithm is programmed in $\mathrm{C}$, which allows it to process full-volume scans in near-real time $(<20 \mathrm{~s}$ on the full MeteoSwiss radar volume, depending on the data coverage and complexity of the dealiasing problem). While this is considerably slower than the previous implementation of $4 \mathrm{DD}(\sim 3 \mathrm{~s}$ on the full MeteoSwiss radar volume), the improvements are considerable and results are still delivered within a timely manner. Within the operational framework, R2D2 depends on secondary algorithms, such as the clutter removal algorithm and the VAD algorithm and in its current form cannot be isolated from the operational chain. However, in general, the dealiasing procedure is applicable to any Doppler radar that yields a regular data stream of PPIs at constant elevations.

\section{Results}

\section{a. Error analysis}

To quantify the performance differences of several dealiasing algorithm versions, we utilize the data produced by the COSMO forward radar operator (Wolfensberger and Berne 2018) to work in a controlled framework. A similar approach was applied in Altube et al. (2017) to quantify improvements in velocity processing.

The main advantage of utilizing simulated data here is the availability of the true wind field and the ability to process a large number of time steps. Whereas manual error assessment has been often used in past studies (e.g., James and Houze 2001), it does not guarantee the correct unfolding of the aliased velocity field, especially in turbulent situations. It also strongly constrains the number of elevations that can be processed.

As COSMO-1's resolution is restricted to $1.1 \mathrm{~km}$, it cannot represent very localized shear phenomena and produces a smoother field than is generally observed in reality. Moreover, it does not contain the noise generally present in radar 
measurements. To add noise to the field and increase the likelihood of small-scale shear, Gaussian noise with a standard deviation of $1 \mathrm{~m} \mathrm{~s}^{-1}$ is added to the aliased radar operator data.

When adding the environmental wind information derived from COSMO-1 in this framework, the wind naturally matches better than in reality. Even considering that the first guess field is smoothed and the radar operator has added noise, the agreement is disproportionally high. Together with the shortcoming of smoothness of the wind field, the statistics computed here are to be evaluated qualitatively. Additional checks are performed on the observed radar data of the same cases to confirm the performance obtained within the radar operator framework.

To quantify the performance, the percentage of erroneous gates in relation to all valid gates containing data, is computed. Empty gates are disregarded, as well as deleted gates. Data deletion only occurs in noise suppression and is not a result of dealiasing failure.

\section{b. R2D2 evaluation using COSMO-1 simulated radar data}

Since recursion requires more computation time, especially in challenging dealiasing situations, the algorithm is also evaluated without iterations, hereafter Region-based Doppler Dealiasing (RD2). Figure 5 shows the performance of the different dealiasing algorithms for each evaluated meteorological event. The percentage of erroneous gates among all valid gates is shown. Whereas the overall percentage is low $(<2 \%)$, it is nonetheless desirable to reduce this percentage since errors tend to happen where correct dealiasing is needed most. Our experience has shown that difficult dealiasing situations with both high velocities and large gate-to-gate shear values are of meteorological interest. As evident in Fig. 5, among the cases there is consistent improvement from 4DD using the VAD to R2D2 using COSMO-1. In all cases, R2D2 with COSMO-1 yields the lowest error percentage. Additionally, there is a significant improvement in all algorithm versions when using COSMO- 1 data as reference velocity. Especially when utilizing COSMO-1, RD2 routinely performs worse than 4DD and R2D2. This shows that the iterative process is a requirement to arrive at R2D2's high performance values and justifies the additional computational expense. Where $4 \mathrm{DD}$ processes a volume in $\sim 3 \mathrm{~s}, \mathrm{RD} 2$ requires $\sim 5 \mathrm{~s}$. The iterations cause R2D2 to need $10-20 \mathrm{~s}$ of computation time per volume.

When using the VAD, the case of 11 August 2019 actually shows a decrease in performance from 4DD to RD2 to R2D2. This stems from both RD2 and R2D2 requiring robust guidance information over larger areas. The case study is from the Pointe de la Plaine Morte radar, which is situated at $\sim 3000 \mathrm{~m}$ MSL. At these high altitudes there is only sparse data coverage, particularly in the higher sweeps. Additionally, the case analyzed shows supercellular convection with scattered precipitation cells. The compounding effect of sparse data coverage in low elevations and a lack of data from higher elevations leads to very unreliable VAD estimates. This in turn can lead to mistakes when assigning the Nyquist interval to regions. In RD2, this leads to a fraction of regions being dealiased erroneously. However, in R2D2, the iterative

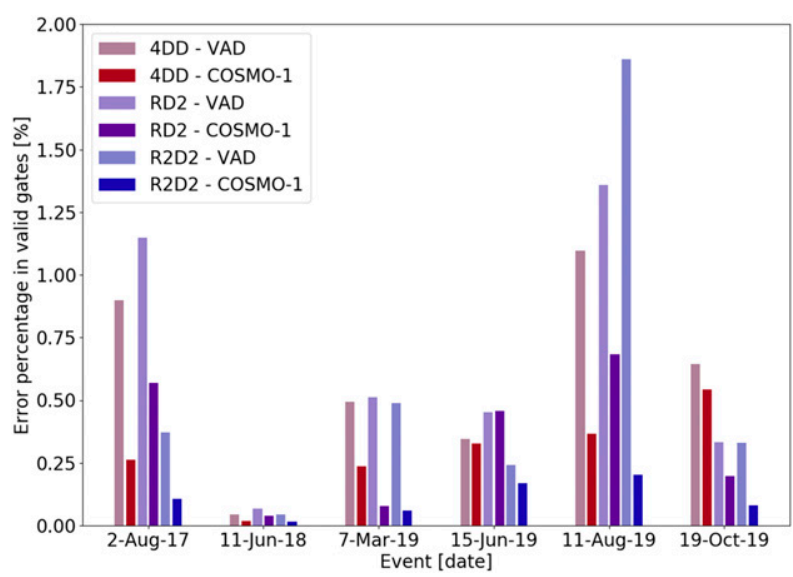

FIG. 5. Error statistics for different dealiasing versions, separated by evaluated event: percentage of erroneous gates within all valid gates of a case study, computed on modeled data.

procedure propagates these errors to larger contiguous regions due to a lack of better guidance data. When using COSMO-1, we can see that this issue does not occur, emphasizing the importance of supplemental wind information in situations of sparse data coverage.

The quantitative error analysis shows that using COSMO1 to provide environmental wind information and applying our region-based, recursive dealiasing approach both contribute to substantial improvements in dealiasing accuracy. Across all analyzed cases, COSMO-1 reduces the number of erroneous gates by $\sim 50 \%$. Moreover, adding the switch from 4DD to R2D2 further reduces the number of erroneous gates by $\sim 50 \%$, yielding a final improvement over all cases of $\sim 75 \%$.

The improvements of COSMO-1 and R2D2 are cumulative, as they target different sources of errors in dealiasing. The use of COSMO-1 targets initialization issues in isolated cells at far ranges. The VAD does not represent these areas very well, as it assumes spatial homogeneity, which can lead to large regions being dealiased incorrectly, even in R2D2. Additionally, it requires a high data coverage to produce robust results, which is generally not the case when dealing with isolated cells. Especially with cells first entering the radar range, there is also no previously dealiased sweep to derive information from. COSMO-1 represents the general flow well enough to initialize these cells in the correct Nyquist interval, resulting in the significant improvement seen here.

R2D2 targets several different issues. The region-based approach reduces the influence of single gates, basing dealiasing decisions on a larger consensus. By checking the deviations from the general decision within each region, an additional error check is introduced to avoid large spatial extents of errors. The iterative approach additionally reduces dealiasing errors in regions, as the regions start merging with increasing iterations, as velocity folds are removed. Larger regions are more robust, as they can be verified with more guidance data. By mitigating spatial dealiasing to small buffer zones, the potential to spread errors this way is also strongly 
reduced. This allows a stronger relaxation of thresholds for spatial dealiasing, which facilitates retrieving the correct velocity values in situations when the gate-to-gate shear is on the order of the Nyquist velocity.

The convective weather situations benefit most from the improvements in the R2D2 scheme. Localized turbulence is targeted more specifically and error propagation is strongly reduced. COSMO-1 equally aids stratiform and convective situations, as it plays a crucial role in correctly initializing dealiasing of data appearing at the outer ranges of the radars.

\section{c. R2D2 on operational radar data}

To verify the general trends observed in the radar operator framework, the same case studies are processed with operational radar data. Here, we can inspect the performance of R2D2 on noisier data that generally contains higher shear values. Additionally, the COSMO-1 data no longer provide a perfect match and we can investigate its benefit over the operational VAD. A visual inspection of the results of the case studies generally confirms the trends observed in the radar operator. R2D2 and COSMO-1 individually contribute to substantial improvements and target different areas of issues, thus yielding the optimal result, when combined. The following examples focus on showcasing the improvement gained from R2D2 over 4DD, as well as the improvement from using COSMO-1 over VAD estimates.

The example shown in Figs. 6 and 7 illustrates the differences between 4DD and R2D2 in a case with supercellular convection. The velocity data at 0000 UTC 2 August 2017 at $1.6^{\circ}$ elevation are shown. Figure 6 a depicts the radar reflectivity and the corresponding raw velocity is illustrated in Fig. 6 b. Figures $6 \mathrm{c}$ and $6 \mathrm{~d}$ show the different first guess fields derived from the VAD and COSMO-1. Additionally, Figs. 6e and $6 f$ show the 4D guidance data from the previous and above elevations, here dealiased with R2D2 using COSMO-1. We can clearly see that the VAD did not perform correctly, due to poor data coverage in the vicinity of the radar. The radial velocity data over the entire sweep are thus vastly underestimated. Moreover, the VAD cannot be produced for all altitude levels, resulting in large data gaps between available values. These issues can be seen in Fig. 6c, as the data are interpolated over large stretches, resulting in large areas of identical information, e.g., from 0 to $70 \mathrm{~km}$ range and from 70 to $160 \mathrm{~km}$ range. As can be seen in Fig. 6d, the first guess field derived from COSMO-1 shows a much smoother data transition, but also generally higher velocity values. It also represents the spatial heterogeneity of a wind field in an orographic environment much better.

Figure 7a shows the results of applying 4DD with the VAD, and Fig. 7b shows the results of 4DD with COSMO-1, revealing that there is a large spread of dealiasing error that can be traced back to an area of unresolved shear at $50 \mathrm{~km}$ range and $320^{\circ}$ azimuth. Without any additional constraints, the error can propagate through spatial dealiasing and negatively affect the subsequent sweeps. Figures. 7c and 7d show R2D2's results with the VAD and COSMO-1, respectively. Here, we clearly see the rotational structure of the mesocyclone at $50 \mathrm{~km}$ range and $320^{\circ}$ azimuth. There are still errors detected (e.g., at $60 \mathrm{~km}$ range and $5^{\circ}$ azimuth), however, they are constrained to much smaller areas. Overall the main dynamical features are retrieved even in this extreme, high-shear environment. Figures $7 \mathrm{e}$ and $7 \mathrm{f}$ depict the identified buffer zones during the first and last, here third, iteration. The merging of regions over folds, while preserving the buffer zones over sheared areas, is clearly visible. This decrease in separate regions also demonstrates an increase in dealiasing robustness throughout the iterations. The first iteration (Fig. 7e) depends on correct dealiasing decisions for many more groups of data, whereas the third iteration (Fig. 7f) mainly needs to solve the separate precipitation regions, before filling in the shear zones with spatial dealiasing.

When comparing the results of dealiasing with the VAD versus dealiasing with COSMO-1, it is noticeable that this only affects few areas. Both algorithms rely on the first guess field only as a last resort, reducing the amount of data affected. Nonetheless, we can see that along $45^{\circ}$ azimuth, several smaller cells benefit from the use of COSMO-1 and appear to be dealiased correctly in Figs. $7 \mathrm{~b}$ and $7 \mathrm{~d}$.

Figures 8 and 9 showcase a dealiasing example at 1820 UTC 11 June 2018 at $1.6^{\circ}$ elevation. Figures $8 \mathrm{a}$ and $8 \mathrm{~b}$ depict the radar reflectivity and raw velocity. Figure $8 \mathrm{c}$ shows the first guess field derived from the VAD, whereas Fig. 8d is derived from COSMO-1. We can see that the data coverage is again somewhat localized, resulting in difficulties when deriving the VAD. Here, the VAD was only produced at one altitude relevant to this elevation. In Fig. $8 \mathrm{c}$, we can see that the limited VAD results in a single estimate of velocity magnitude and direction for all ranges. On the other hand, the first guess field from COSMO-1 (see Fig. 8d) provides velocity values that are much higher and spatially heterogeneous. Figures $8 \mathrm{e}$ and $8 \mathrm{f}$ additionally show the 4D guidance data obtained from the previous and above elevations. In Figs. 9a and $9 \mathrm{~b}$, the data are dealiased with 4DD using the VAD and COSMO-1. These figure panels clearly illustrate that the use of COSMO-1 data instead of a VAD as supplemental wind information can dramatically improve algorithm performance. COSMO-1 mitigates errors that occur mainly at far ranges, but generally affect large areas and is thus a substantial improvement. Figures 9c and 9d depict R2D2's performance using the VAD and COSMO-1. In this case, the region-based approach already resolved the major error using the VAD at $100-160 \mathrm{~km}$ and $220^{\circ}-260^{\circ}$. The addition of COSMO-1 further contributes to minor improvements along $45^{\circ}$ azimuth. In this case, an isolated convective cell is developing at $60 \mathrm{~km}$ range but is not well represented by the VAD, while the COSMO-1 data lead to a reliable interpretation of the Doppler velocity in that location. Figures 9e and 9 f also show the progress of region merging between the first and last (third) iteration. Here we can also see that even with the velocity field appearing more homogeneous, there are still velocity shears present that exceed $0.8 v_{\mathrm{NYQ}}$ within neighboring gates. These shear zones remain flagged as buffer zones in the last iteration of R2D2. This shows the challenging nature of dealiasing in the context of low Nyquist velocities. 
a) reflectivity, [dBz]

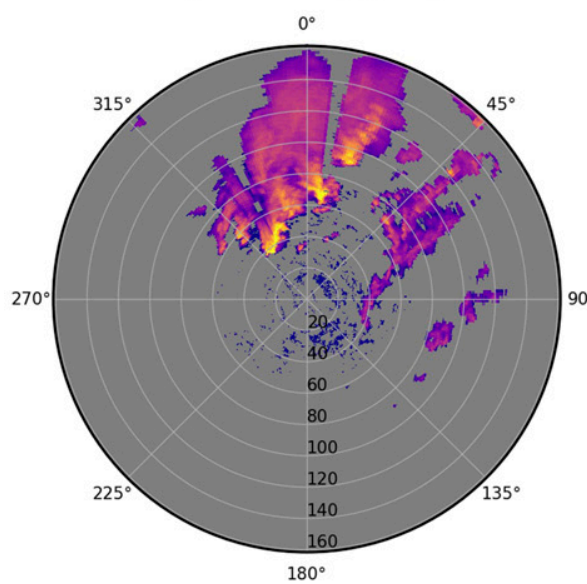

c) VAD, $\left[\mathrm{m} \mathrm{s}^{-1}\right]$

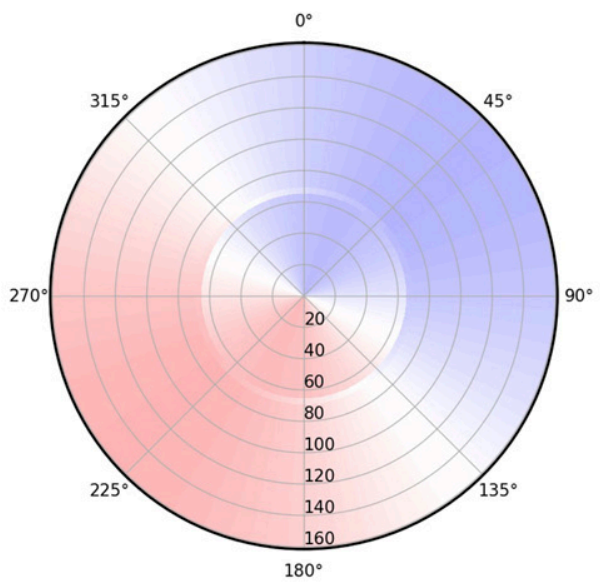

e) previous sweep, $\left[\mathrm{m} \mathrm{s}^{-1}\right]$

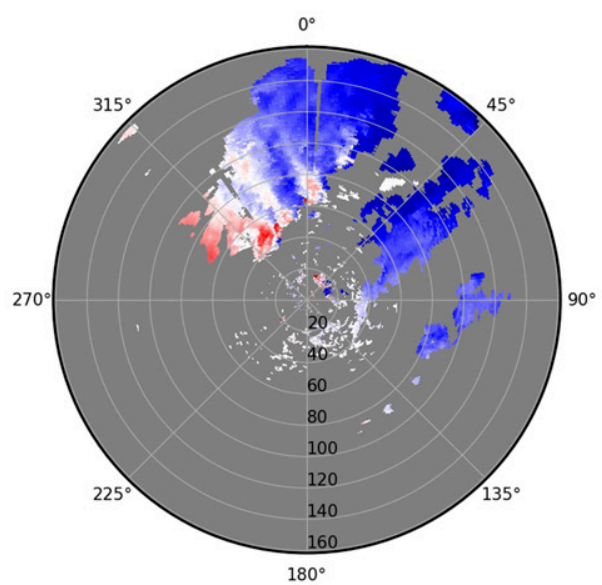

b) raw velocity, [ $\left.\mathrm{m} \mathrm{s}^{-1}\right]$
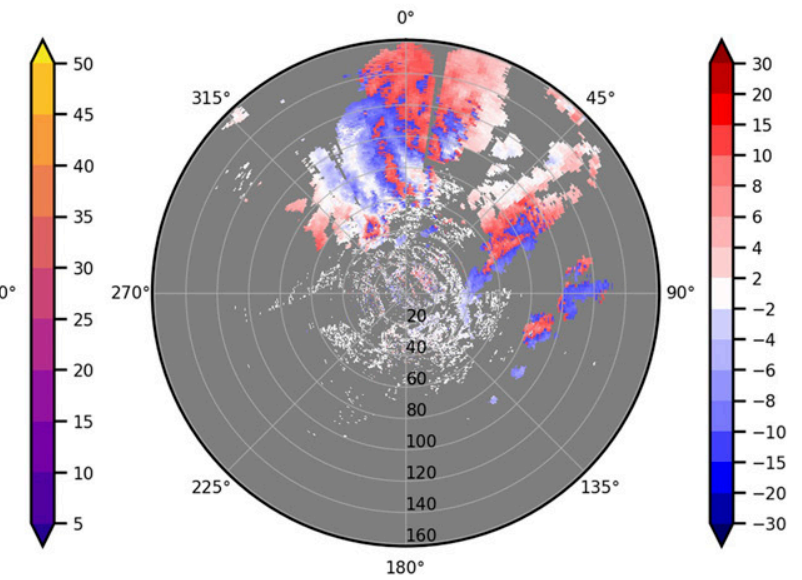

d) COSMO-1, $\left[\mathrm{m} \mathrm{s}^{-1}\right]$

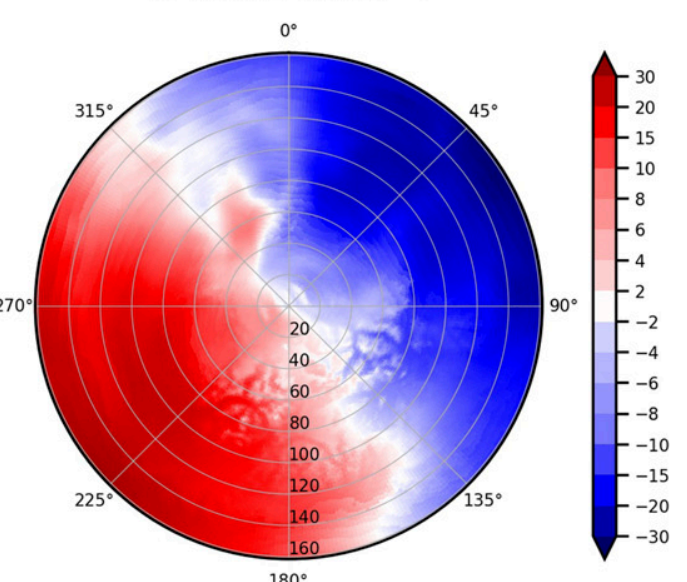

f) upper sweep, [m s $\left.{ }^{-1}\right]$

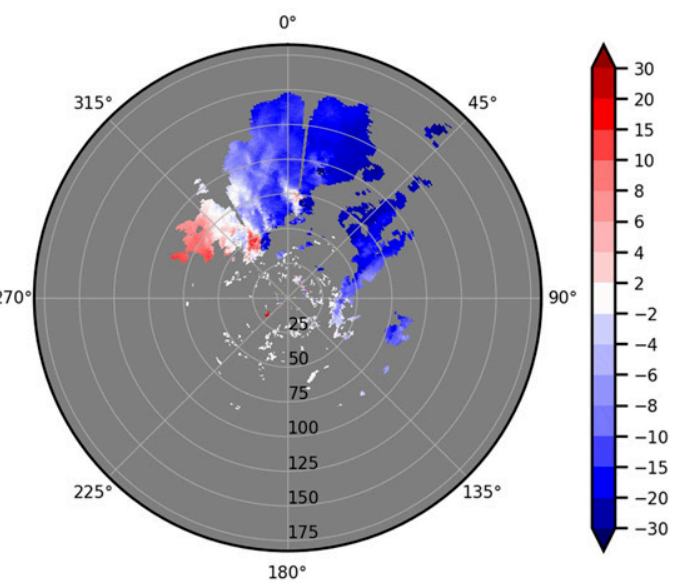

FIG. 6. Case study at 0000 UTC 2 Aug 2017, Albis radar, $1.6^{\circ}$ elevation, $v_{\mathrm{NYQ}}=12.4 \mathrm{~m} \mathrm{~s}^{-1}$ : (a) reflectivity, (b) raw velocity, (c) VAD, and (d) COSMO-1 estimates; (e),(f) guidance data from the previous and upper dealiased sweeps.

Our tests also reveal that dealiasing results are often forgiving of misplaced convective cells. When using COSMO-1 data whose valid time had a $3 \mathrm{~h}$ offset from the observed radar data, we obtained largely the same dealiasing results, with very few deviations. Since COSMO-1 grids are interpolated and used as a last resort in the dealiasing process, we postulate that obtaining the precise location of deep convection in the model is generally not necessary. Moreover, mesoscale 
a) $4 D D$ - VAD, $\left[\mathrm{m} \mathrm{s}^{-1}\right]$

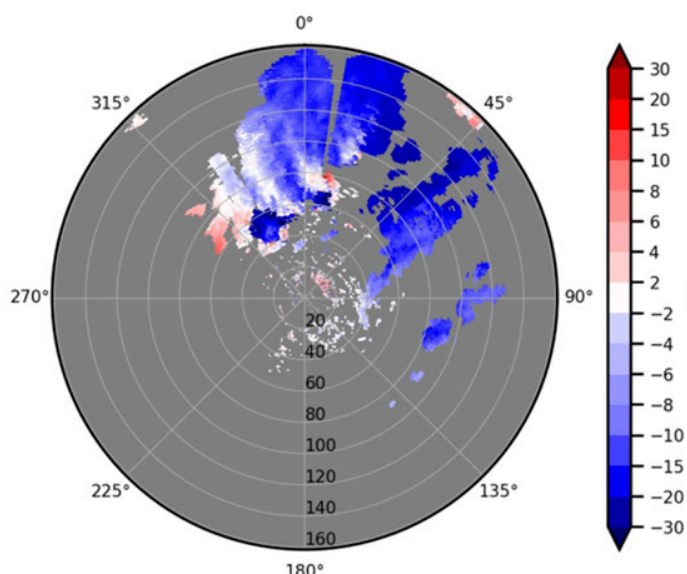

c) R2D2 - VAD, $\left[\mathrm{m} \mathrm{s}^{-1}\right]$

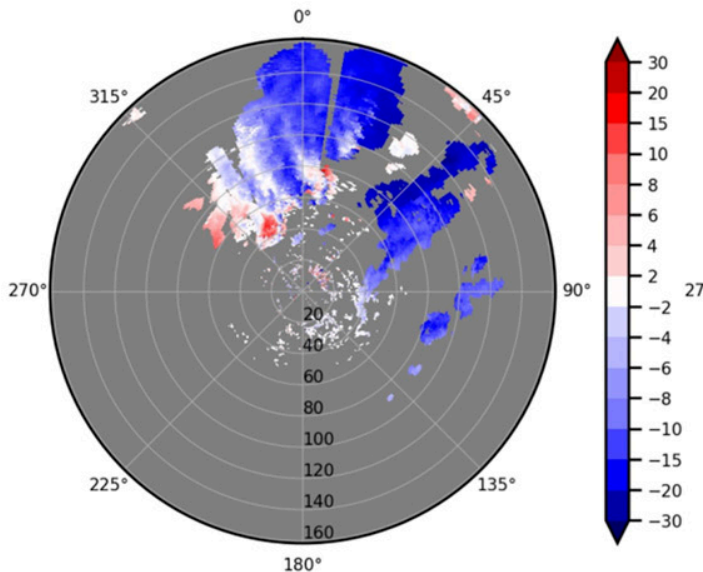

e) buffer zones first iteration

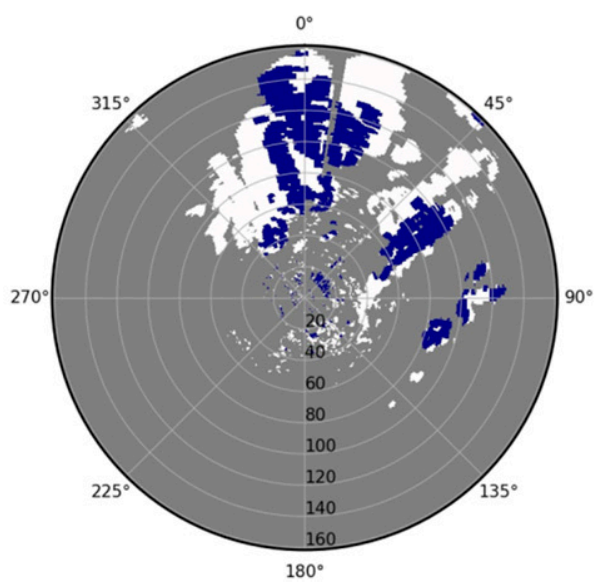

b) 4DD - COSMO-1, $\left[\mathrm{m} \mathrm{s}^{-1}\right]$

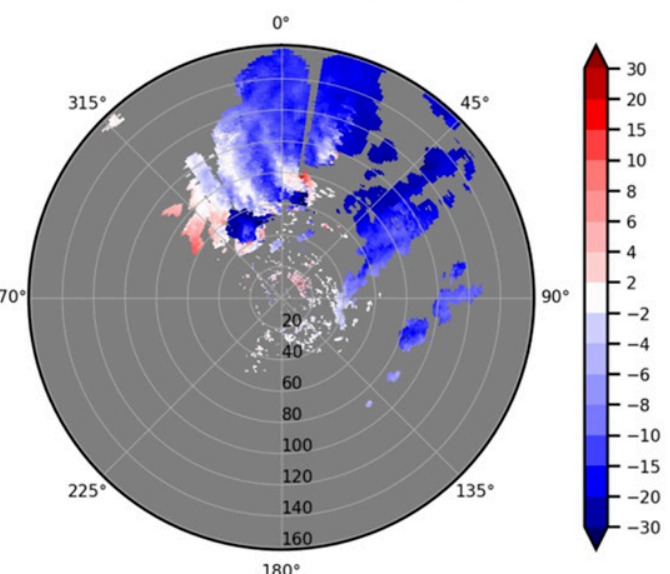

d) R2D2 - COSMO-1, $\left[\mathrm{m} \mathrm{s}^{-1}\right]$

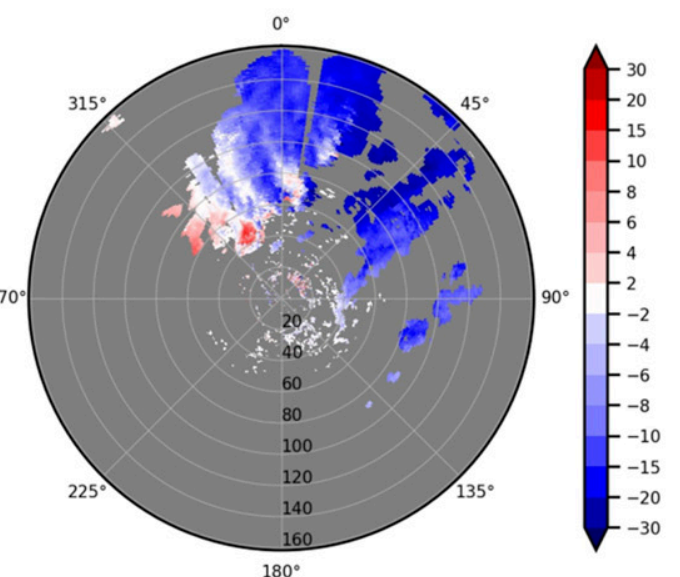

f) buffer zones last iteration

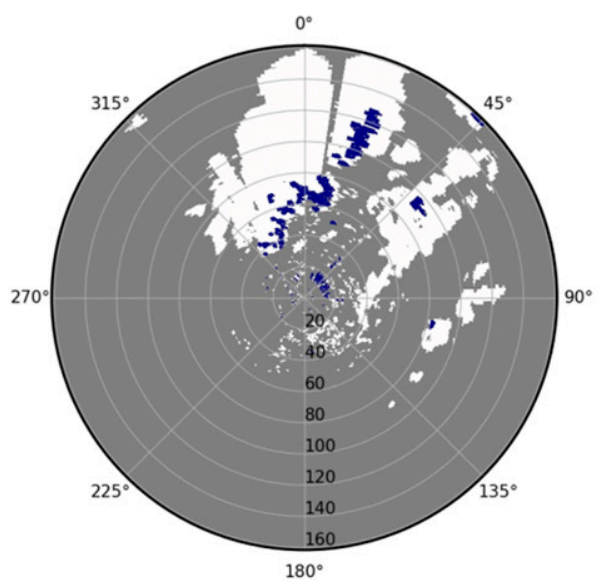

FIG. 7. Dealiasing example at 0000 UTC 2 Aug 2017, Albis radar, $1.6^{\circ}$ elevation, $v_{\mathrm{NYQ}}=12.4 \mathrm{~m} \mathrm{~s}^{-1}$ : dealiasing outcomes of (a) 4DD with the VAD, (b) 4DD with COSMO-1, (c) R2D2 with the VAD, and (d) R2D2 with COSMO-1; (e),(f) buffer zones (blue) of first and last (third) iterations of R2D2 with COSMO-1. 
a) reflectivity, [dBz]

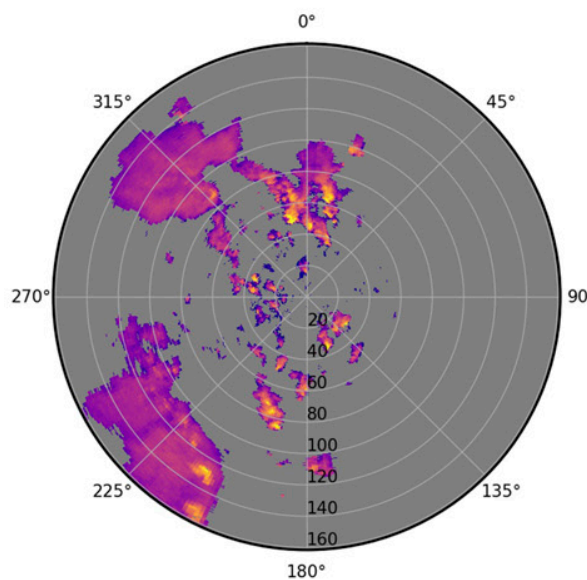

c) VAD, $\left[\mathrm{m} \mathrm{s}^{-1}\right]$

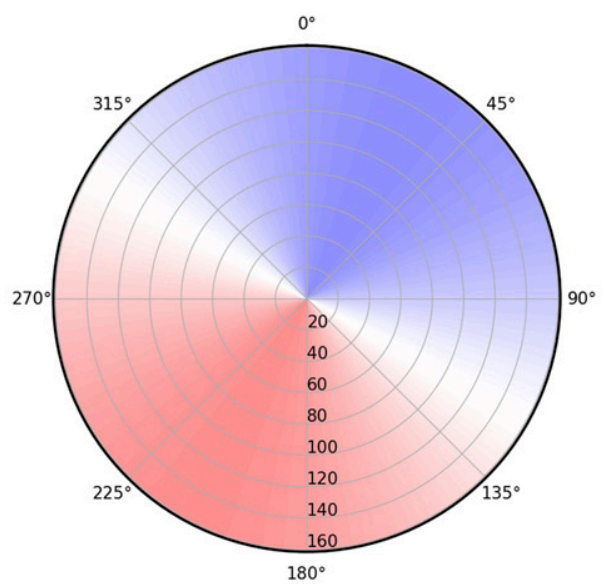

e) previous sweep, [ $\left.\mathrm{m} \mathrm{s}^{-1}\right]$

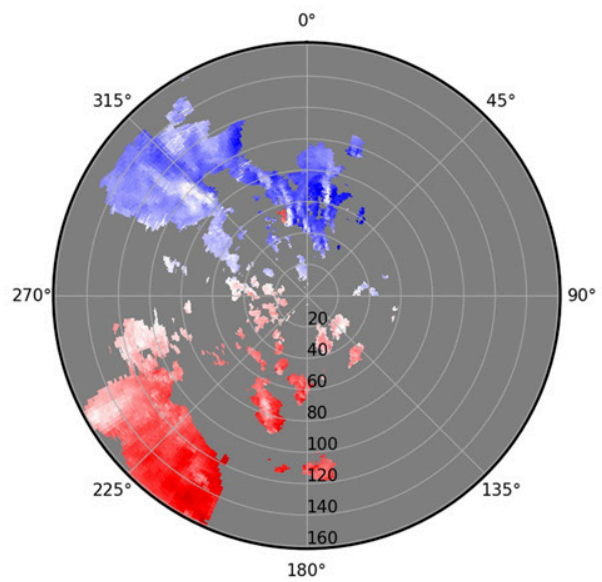

b) raw velocity, $\left[\mathrm{m} \mathrm{s}^{-1}\right]$

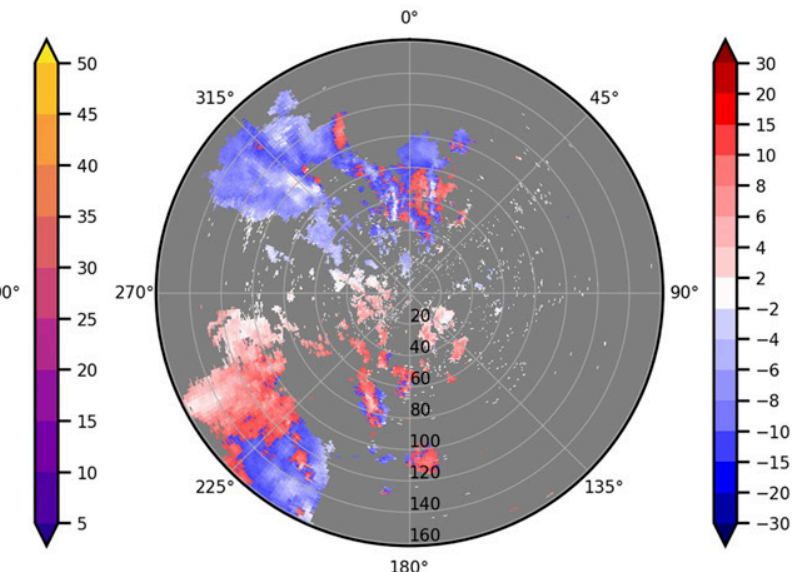

d) COSMO-1, $\left[\mathrm{m} \mathrm{s}^{-1}\right]$

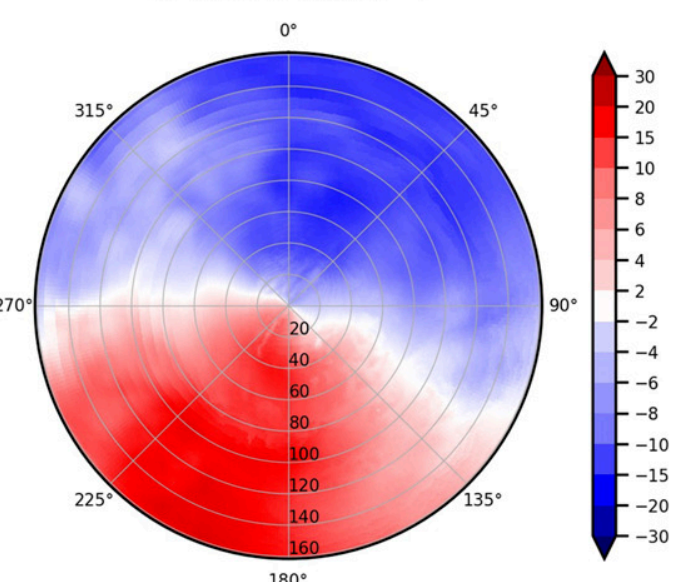

f) upper sweep, [m s $\left.\mathrm{m}^{-1}\right]$
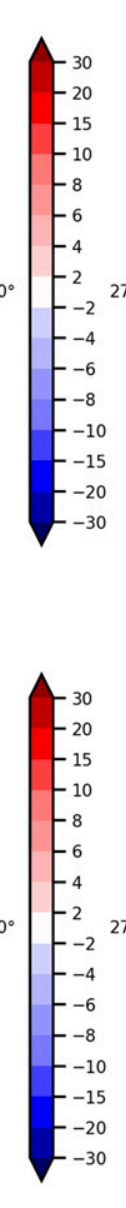

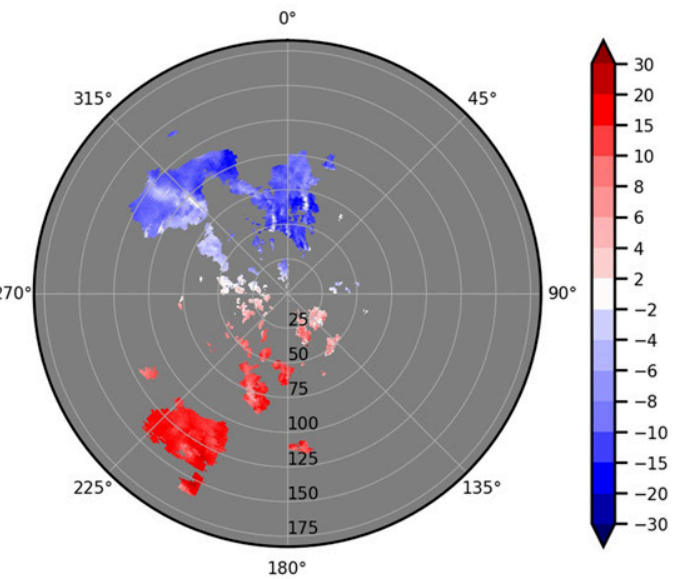

FIG. 8. Case study at 1815 UTC 11 Jun 2018, La Dôle radar, $1.6^{\circ}$ elevation, $v_{\mathrm{NYQ}}=12.4 \mathrm{~m} \mathrm{~s}^{-1}$ : (a) reflectivity, (b) raw velocity, (c) VAD, and (d) COSMO-1 estimates; (e),(f) guidance data from the previous and upper dealiased sweeps. 
a) $4 D D$ - VAD, $\left[\mathrm{m} \mathrm{s}^{-1}\right]$

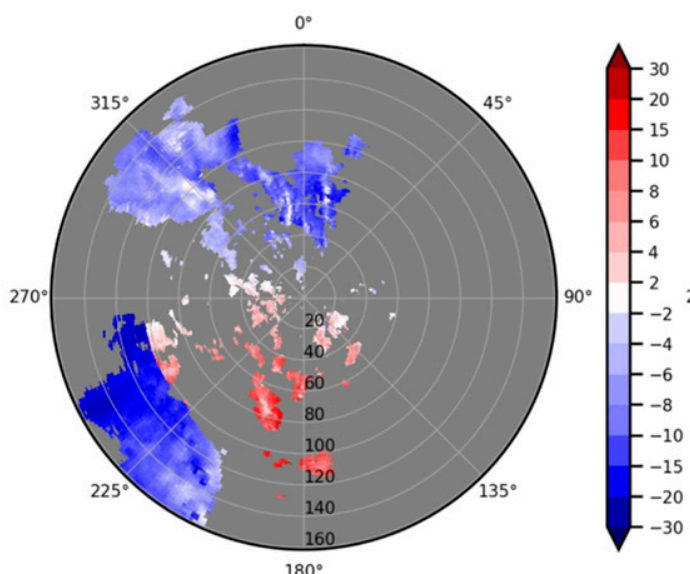

c) R2D2 - VAD, $\left[\mathrm{m} \mathrm{s}^{-1}\right]$

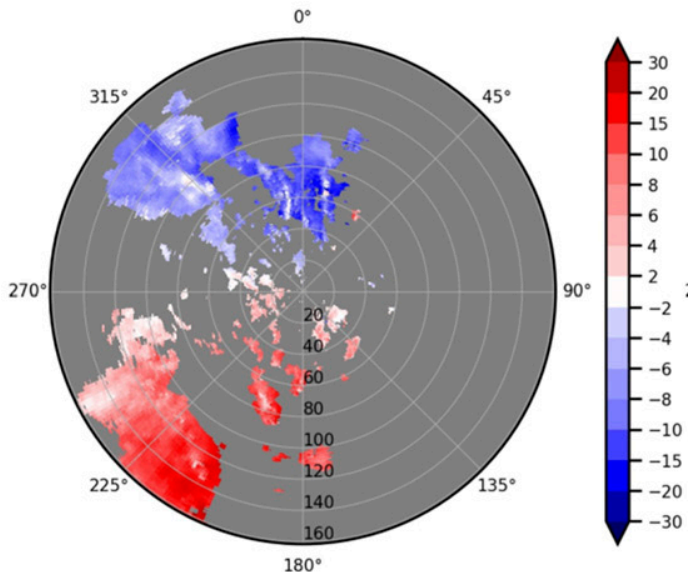

e) buffer zones first iteration

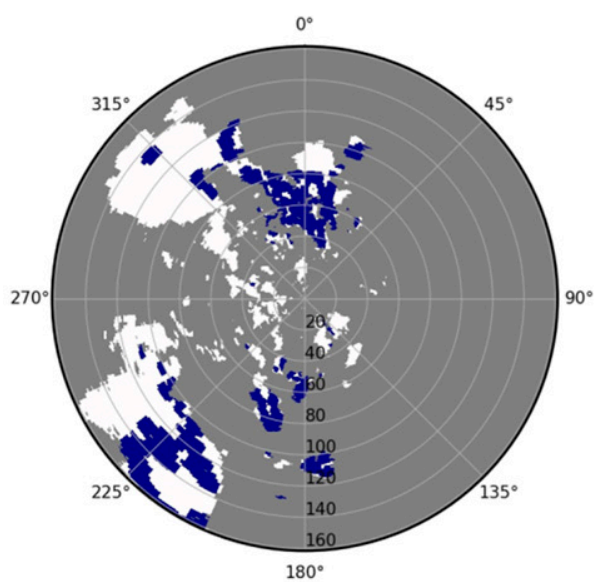

b) 4DD - COSMO-1, $\left[\mathrm{m} \mathrm{s}^{-1}\right]$

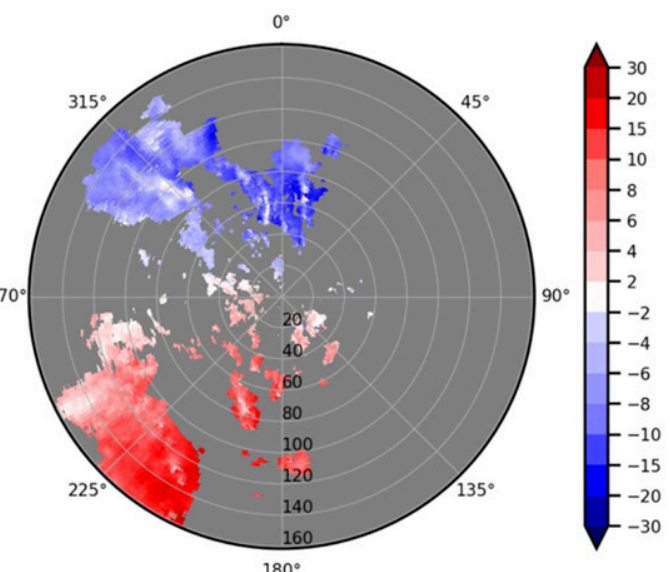

d) R2D2 - COSMO-1, $\left[\mathrm{m} \mathrm{s}^{-1}\right]$

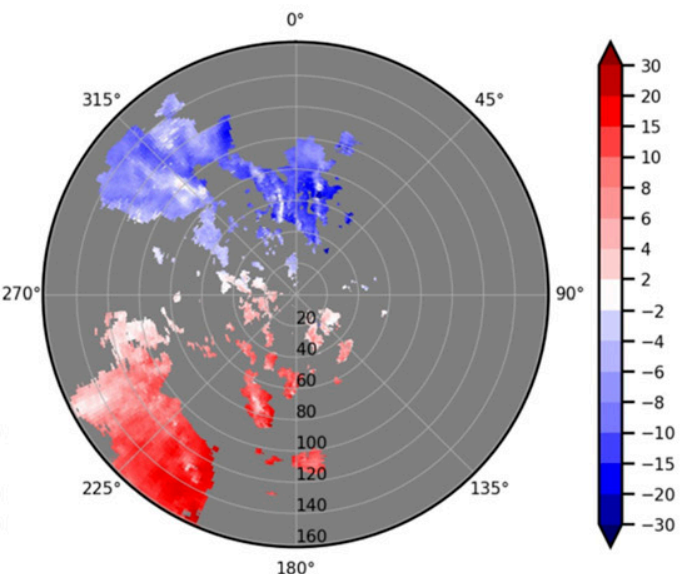

f) buffer zones last iteration

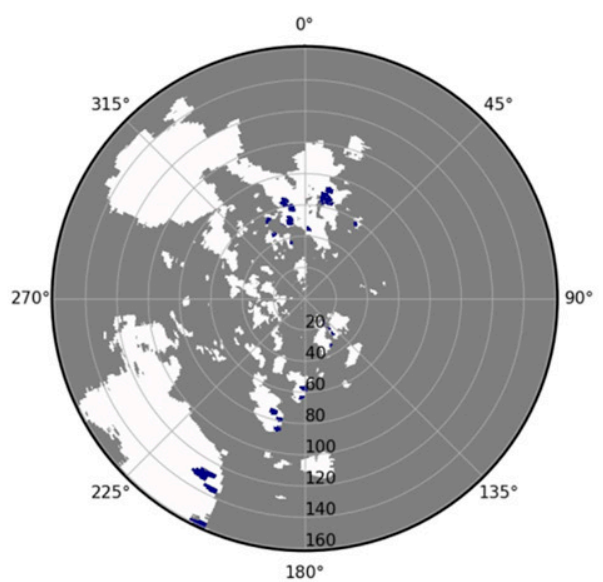

FIG. 9. Dealiasing example at 1815 UTC 11 Jun 2018, La Dôle radar, $1.6^{\circ}$ elevation, $v_{\mathrm{NYQ}}=12.4 \mathrm{~m} \mathrm{~s}^{-1}$ : dealiasing outcomes of (a) 4DD with the VAD, (b) 4DD with COSMO-1, (c) R2D2 with the VAD, and (d) R2D2 with COSMO-1; (e),(f) buffer zones (blue) of first and last (third) iterations of R2D2 with COSMO-1. 
forecast grids, as opposed to accurate mesoscale analyses of convective precipitation, would be more than sufficient and may be the only option for dealiasing in an operational context.

\section{Conclusions}

The novel dealiasing algorithm R2D2 adequately achieves our objectives of region-based initialization, 4D continuity, mitigated error propagation, improved environmental wind estimates, and suitability for operational implementation in challenging dealiasing situations.

Improved algorithm design and environmental wind estimates contribute to substantial improvements in various meteorological situations over the existing Swiss operational algorithm. The best results are achieved when combining COSMO-1's first guess field with R2D2. The improvements are robust in a variety of convective and stratiform case studies. The most challenging situations occur, however, when the velocity differences between two gates exceed the Nyquist velocity. This often leads to dealiasing errors; however, R2D2 contains these errors to small areas.

Our analysis included cases that portrayed different but challenging wind situations, including high-shear environments of supercells and mesoscale convective systems, as well as the high wind speeds and wind direction changes associated with a strong cold front passage. The performance increase of R2D2 is robust across all cases and therefore suitable for a general, operational application. Completing the dealiasing cycle once is a computationally efficient procedure. The iterative approach takes accordingly longer, depending on how many iterations are needed to find the optimum solution. R2D2 requires more processing time than $4 \mathrm{DD}$, especially in challenging situations. Nonetheless, the data can be processed in near-real time and the procedure is suitable for operational implementation.

The dealiasing procedure in R2D2 is generally suited for a continuous stream of radar data measured in a succession of PPIs. While it processes data top-down, the measurements can generally proceed in any order, as long as the assumption of data continuity between elevations and time steps are still valid.

Acknowledgments. This research was made possible by a three-way partnership between MeteoSwiss, EPFL, and Embry-Riddle Aeronautical University. The data used in the case study analyses are available upon request. Due to the proprietary nature of the software the code cannot be made available.

\section{REFERENCES}

Altube, P., J. Bech, O. Argemi, T. Rigo, N. Pineda, S. Collis, and J. Helmus, 2017: Correction of dual-PRF Doppler velocity outliers in the presence of aliasing. J. Atmos. Oceanic Technol., 34, 1529-1543, https://doi.org/10.1175/JTECH-D-16-0065.1.

Bergen, W. R., and S. C. Albers, 1988: Two- and three-dimensional de-aliasing of Doppler radar velocities. J. Atmos. Oceanic Technol., 5, 305-319, https://doi.org/10.1175/1520-0426(1988) $005<0305$ :TATDDA $>2.0 . \mathrm{CO} ; 2$.
Bundesamt für Meteorologie und Klimatologie MeteoSchweiz, 2018: Das Schweizer Wetterradarnetz. MeteoSwiss, accessed 12 December 2019, 4 pp., https://www.meteoswiss.admin.ch/ content/dam/meteoswiss/de/Mess-und-Prognosesysteme/ Atmosphaere/Radarnetz/doc/20170830_Radarnetz_DE_ einzeln.pdf.

Chang, P.-L., W.-T. Fang, P.-F. Lin, and M.-J. Yang, 2019: A vortex-based Doppler velocity dealiasing algorithm for tropical cyclones. J. Atmos. Oceanic Technol., 36, 1521-1545, https://doi.org/10.1175/JTECH-D-18-0139.1.

de Morsier, G., O. Fuhrer, and M. Arpagaus, 2012: Challenges for a new $1 \mathrm{~km}$ non-hydrostatic model over the Alpine area. 15th Int. Conf. on Mountain Meteorology, Steamboat Springs, CO, Amer. Meteor. Soc., 3, https://ams.confex.com/ams/ 15MountMet/webprogram/Paper210187.html.

Eilts, M. D., and S. D. Smith, 1990: Efficient dealiasing of Doppler velocities using local environment constraints. J. Atmos. Oceanic Technol., 7, 118-128, https://doi.org/10.1175/15200426(1990)007<0118:EDODVU>2.0.CO;2.

Gabella, M., P. Speirs, U. Hamann, U. Germann, and A. Berne, 2017: Measurement of precipitation in the Alps using dualpolarization C-band ground-based radars, the GPM spaceborne Ku-band radar, and rain gauges. Remote Sens., 9, 1147, https://doi.org/10.3390/RS9111147.

Germann, U., 1999: Vertical wind profile by Doppler radars. MAP Newsletter, No. 11, MeteoSwiss, Zurich, Switzerland, 6-7.

, and J. Joss, 2004: Operational measurement of precipitation in mountainous terrain. Weather Radar: Principles and Advanced Applications, P. Meischner, Ed., Springer, 52-77.

—-, M. Boscacci, M. Gabella, and M. Sartori, 2015: Peak performance: Radar design for prediction in the Swiss Alps. Meteorological Technology International, April 2015, UKIP Media \& Events, 42-45, www.ukimediaevents.com/ publication/574f8129/44.

- -1, -, and M. Schneebeli, 2016a: Weather radar in Switzerland. From Weather Observations to Atmospheric and Climate Sciences in Switzerland, S. Willemse and M. Furger, Eds., vdf Hochschulverlag AG, 165-188.

_, J. Figueras i Ventura, M. Gabella, A. M. Hering, I. Sideris, and B. Calpini, 2016b: Triggering innovation: The latest MeteoSwiss Alpine weather radar network. Meteorological Technology International, April 2015, UKIP Media \& Events, 62-65, www.ukimediaevents.com/publication/2d183b22/64.

He, G., G. Li, X. Zou, and P. S. Ray, 2012: Applications of a velocity dealiasing scheme to data from the China New Generation Weather Radar System (CINRAD). Wea. Forecasting, 27, 218230, https://doi.org/10.1175/WAF-D-11-00054.1.

Helmus, J. J., and S. M. Collis, 2016: The Python ARM Radar Toolkit (Py-ART), a library for working with weather radar data in the Python Programming Language. J. Open Res. Soft., 4, e25, https://doi.org/10.5334/jors.119.

James, C. N., and R. A. J. Houze, 2001: A real-time four-dimensional Doppler dealiasing scheme. J. Atmos. Oceanic Technol., 18, 1674-1683, https://doi.org/10.1175/1520-0426(2001)018<1674: ARTFDD $>2.0 . \mathrm{CO} ; 2$.

Jiang, Y., and Q. Xu, 2016: Adaptive dealiasing for Doppler velocities scanned from hurricanes and typhoons. J. Atmos. Oceanic Technol., 33, 1931-1947, https://doi.org/10.1175/ JTECH-D-15-0146.1.

Liang, X., Y. Xie, J. Yin, Y. Luo, D. Yao, and F. Li, 2019: An IVAP-based dealiasing method for radar velocity data quality control. J. Atmos. Oceanic Technol., 36, 2069-2085, https:// doi.org/10.1175/JTECH-D-18-0216.1. 
Lim, E., and J. Sun, 2010: A velocity dealiasing technique using rapidly updated analysis from a four-dimensional variational Doppler radar data assimilation system. J. Atmos. Oceanic Technol., 27, 1140-1152, https://doi.org/10.1175/2010JTECHA1300.1.

Louf, V., A. Protat, R. C. Jackson, S. M. Collis, and J. Helmus, 2020: UNRAVEL: A robust modular velocity dealiasing technique for Doppler radar. J. Atmos. Oceanic Technol., 37, 741-758, https://doi.org/10.1175/JTECH-D-19-0020.1.

Markowski, P., and Y. Richardson, 2010: Mesoscale Meteorology in Midlatitudes. Wiley-Blackwell, $430 \mathrm{pp}$.

MeteoSwiss, 2020: Clutter in der wetterradartechnik. MeteoSwiss, accessed 14 July 2020, https://www.meteoschweiz.admin.ch/home/ aktuell/meteoschweiz-blog/meteoschweiz-blog.subpage.html/ de/data/blogs/2020/6/clutter_in_der_wetterradartechnik.html.

Tabary, P., G. Scialom, and U. Germann, 2001: Real-time retrieval of the wind from aliased velocities measured by Doppler radars. J. Atmos. Oceanic Technol., 18, 875-882, https://doi.org/ 10.1175/1520-0426(2001)018<0875:RTROTW $>2.0$. CO;2.

Witt, A., and R. A. Brown, 2009: Performance of a new velocity dealiasing algorithm for the WSR-88D. 34th Conf. on Radar Meteorology, Williamsburg, VA, Amer. Meteor. Soc., P4.8, https://ams.confex.com/ams/pdfpapers/155951.pdf.

Wolfensberger, D., and A. Berne, 2018: From model to radar variables: A new forward polarimetric radar operator for COSMO. Atmos. Meas. Tech., 11, 3883-3916, https://doi.org/ 10.5194/amt-11-3883-2018.
Wüest, M., U. Germann, and W. Schmid, 2000: A variational dealiasing technique. Phys. Chem. Earth, 25, 1179-1183, https:// doi.org/10.1016/S1464-1909(00)00175-1.

Xu, Q., and K. Nai, 2017: Mesocyclone-targeted Doppler velocity dealiasing. J. Atmos. Oceanic Technol., 34, 841-853, https:// doi.org/10.1175/JTECH-D-16-0170.1.

- - , and L. Wei, 2010: Fitting VAD winds to aliased Doppler radial-velocity observations: A global minimization problem in the presence of multiple local minima. Quart. J. Roy. Meteor. Soc., 136, 451-461, https://doi.org/10.1002/ qj.589.

,,,--- P. Zhang, S. Liu, and D. Parrish, 2011: A VADbased dealiasing method for radar velocity data quality control. J. Atmos. Oceanic Technol., 28, 50-62, https://doi.org/ 10.1175/2010JTECHA1444.1.

,-- C. Karstens, T. Smith, and Q. Zhao, 2013: Improved Doppler velocity dealiasing for radar data assimilation and storm-scale vortex detection. Adv. Meteor., 2013, 562386, https://doi.org/10.1155/2013/562386.

Yuan, Y., P. Wang, D. Wang, and J. Shi, 2020: A velocity dealiasing scheme based on minimization of velocity differences between regions. Adv. Meteor., 2020, 6157636, https://doi.org/10.1155/ 2020/6157636.

Zhang, J., and S. Wang, 2006: An automated 2D multipass Doppler radar velocity dealiasing scheme. J. Atmos. Oceanic Technol., 23, 1239-1248, https://doi.org/10.1175/JTECH1910.1. 